\title{
Cost-Effectiveness Analysis for the Treatment of Hyperphosphatemia in Predialysis Patients: Calcium-Based versus Noncalcium-Based Phosphate Binders
}

\author{
B. L. Goh $\mathbb{D},{ }^{1}$ A. Soraya, ${ }^{2}$ A. Goh, ${ }^{2}$ and K. L. Ang $\mathbb{C}^{1}$ \\ ${ }^{1}$ Clinical Research Centre, Serdang Hospital, Kajang 43000, Malaysia \\ ${ }^{2}$ Azmi Burhani Consulting, Petaling Jaya 47820, Malaysia \\ Correspondence should be addressed to B. L. Goh; bak.leong@gmail.com
}

Received 27 June 2018; Accepted 2 September 2018; Published 19 September 2018

Academic Editor: Jaime Uribarri

Copyright (C) 2018 B. L. Goh et al. This is an open access article distributed under the Creative Commons Attribution License, which permits unrestricted use, distribution, and reproduction in any medium, provided the original work is properly cited.

\begin{abstract}
Background. Hyperphosphatemia in chronic kidney disease (CKD) patients is often treated with calcium carbonate (CaCO3) despite the fact that $\mathrm{CaCO} 3$ is associated with increased calcium load and potentially increased cardiovascular risk. Alternative treatments with noncalcium-based phosphate binders do not increase the calcium load but are more costly. This study analyzes the cost-effectiveness of sevelamer versus $\mathrm{CaCO} 3$ for the treatment of hyperphosphatemia in stage III-V predialysis CKD patients in Malaysia. Methods. A Markov decision model was adapted to simulate a hypothetical cohort of CKD patients requiring treatment for hyperphosphatemia. Survival was estimated by using efficacy data from the INDEPENDENT-CKD clinical trial. Cost data was obtained from Malaysian studies while health state utilities were derived from literature. Analysis was performed over lifetime duration from the perspective of the Ministry of Health Malaysia with 2013 as reference year. Results. In the base case analysis, sevelamer treatment gained 6.37 life years (5.27 QALY) compared to 4.25 life years (3.54 QALY) with CaCO3. At 3\% discount, lifetime costs were RM159,901 $(\$ 48,750)$ and RM77,139 $(\$ 23,518)$ on sevelamer and CaCO3, respectively. Incremental cost-effectiveness (ICER) of sevelamer versus CaCO3 was RM47,679 $(\$ 14,536)$ per QALY, which is less than the WHO threshold of three times GDP per capita (RM99,395) per QALY. Sensitivity analyses, both using scenario sensitivity analysis and probabilistic sensitivity analysis, showed the result to be robust. Conclusions. Our study finds that sevelamer is potentially cost-effective compared to $\mathrm{CaCO}$, for the treatment of hyperphosphatemia in predialysis CKD III-V. We propose that sevelamer should be an option in the treatment of Malaysian predialysis patients with hyperphosphatemia, particularly those with high calcium load.
\end{abstract}

\section{Introduction}

Treatment of CKD imposes a substantial cost on health care budgets and middle-income countries (MIC) face significant challenges. In Malaysia, a middle-income country, an estimated $9.1 \%$ of the adult population suffered from CKD in 2013 [1] and this number is rising largely due to the increasing number of patients diagnosed with type 2 diabetes in recent years caused by changing lifestyles and dietary habits $[2,3]$. CKD accounted for $27 \%$ of the total US Medicare budget in $2007[4,5]$. In 2005, Malaysia had spent an estimated RM379 million to provide dialysis services for 13,355 patients [6]. By 2016, the number of CKD Vd patients in Malaysia has tripled to $39,711[7]$.
One of the complications that develop in CKD patients is hyperphosphatemia. Studies have shown that elevated serum phosphate increases risk of mortality and cardiovascular disease (CVD) even for patients with early CKD. In fact, high serum phosphate levels are also associated with more rapid decline in renal function [8-10]. Kidney Disease: Improving Global Outcomes (KDIGO) guidelines recommended maintaining serum phosphate in the normal range (0.81 to $1.45 \mathrm{mmol} / \mathrm{l})$ for patients with renal failure, CKD stage III to $\mathrm{V}$ (CKD-ND) [11].

Calcium-based binders (CBBs) such as calcium carbonate $(\mathrm{CaCO} 3)$ are commonly used since they are widely available and cheap. However, clinical studies have shown that the use of oral calcium in the form of supplements or phosphate 
TABLE 1: INDEPENDENT-CKD trial patient characteristics.

\begin{tabular}{lcc}
\hline Patient characteristics & Sevelamer & CaCO3 \\
\hline Subjects (N) & 107 & 105 \\
Mean age (years) & 57.4 & 58.5 \\
Males (\%) & 61 & 61 \\
CKD disease stage & Stage 3-4 & Stage 3-4 \\
Diabetes (\%) & 27 & 29 \\
Hypertension (\%) & 72.9 & 76.1 \\
Baseline creatinine clearance $(\mathrm{CCr})(\mathrm{ml} / \mathrm{min})$ & 31.7 & 32.7 \\
Baseline phosphorus $(\mathrm{mg} / \mathrm{dl})$ & 4.82 & 4.87 \\
Baseline calcium $(\mathrm{mg} / \mathrm{dl})$ & 9.0 & 8.8 \\
Baseline parathyroid hormone $(\mathrm{PTH})(\mathrm{pg} / \mathrm{ml})$ & 200 & 188 \\
\hline
\end{tabular}

binders may lead to arterial calcification and an increased risk of mortality and CVD events [12-15]. The KDIGO guidelines recommended restricting the dose of CBBs for CKD patients in the presence of arterial calcification [11]. The INDEPENDENT-CKD trial which was performed among 212 patients (107 assigned to sevelamer and 105 assigned to $\mathrm{CaCO} 3)$ reported that treatment with sevelamer in CKDND patients was associated with reductions in mortality and dialysis initiation [16]. Despite concerns regarding the longterm safety of $\mathrm{CBBs}$, $\mathrm{CaCO} 3$ is still the first-line treatment for CKD patients in many low and MIC like Malaysia as they cost less than non-CBBs. Data from the Malaysian National Renal Registry indicated that, in 2016, more than 95\% of CKD Vd patients were prescribed with $\mathrm{CaCO} 3$, and only $17 \%$ have a serum phosphate level of less than $1.3 \mathrm{mmol} / \mathrm{l}$, indicating achievement of target based on the KDIGO guidelines was far from satisfactory [7].

There was evidence that non-CBB like sevelamer was cost-effective in two European countries; the value of this information is limited in the local setting due to differences in health systems and cost structures [17-19]. Thus, this study was conducted to determine the cost-effectiveness of sevelamer compared to $\mathrm{CaCO} 3$ for treatment of hyperphosphatemia in CKD-ND patients in Malaysia.

\section{Methods}

We performed an economic evaluation by adapting the previously developed decision-analysis model: the Markov decision model was adapted to simulate a hypothetical cohort of CKD patients requiring treatment for hyperphosphatemia using available Malaysian data [17]. Decision analysis is an approach for decision making taking into account uncertainty and evaluation of the consequences of alternative courses of action in terms of their costs and outcomes. Markov models are a type of decision-analysis model which are used to analyse uncertain processes, such as chronic diseases in which costs and outcomes occur over a long period of time [20]. Markov type decision models have been widely used in cost-effectiveness analysis including the use of sevelamer in dialysis and CKD-ND patients, as well as other chronic kidney disease interventions $[17,21,22]$.

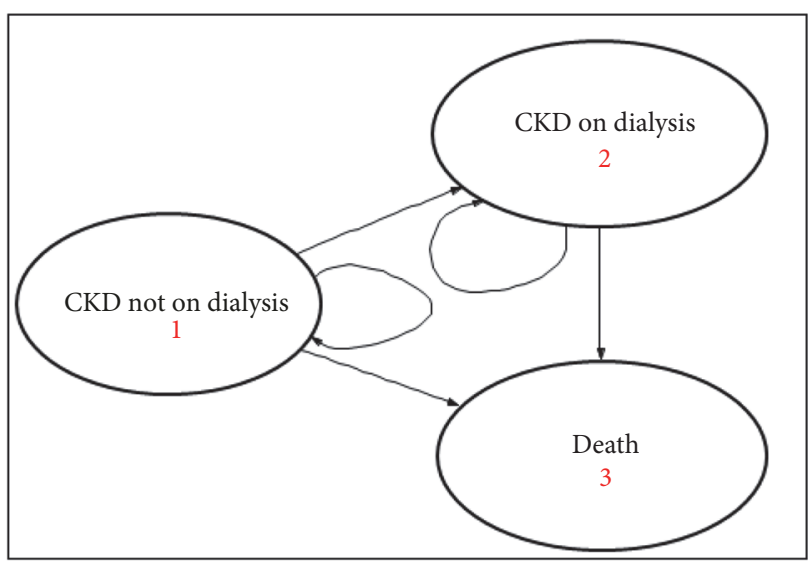

FIGURE 1: State transition diagram of the CLEAR-CKD model.

2.1. Model Overview. The Markov decision model simulates the progression from predialysis CKD to dialysis and death in a hypothetical cohort of CKD-ND patients treated with sevelamer carbonate or $\mathrm{CaCO} 3$ for hyperphosphatemia. The model structure is illustrated in Figure 1. The cohort entered the model in the health state "CKD not on Dialysis" and in subsequent one-year cycles, patients could remain alive without dialysis or transition to dialysis or death. Each cycle spent in the health states of "CKD not on Dialysis" or "CKD on dialysis" was associated with an amount of cost and quality of life. In the event of death, the simulated patient no longer incurs any cost nor gains any heath outcome. The total duration spent by the cohort in each of the health states was aggregated to obtain the total cost and quality-adjusted life years (QALY) accrued on treatment with sevelamer or $\mathrm{CaCO} 3$, respectively. Baseline characteristics of the trial subjects are shown in Table 1 . The data inputs are summarized in Table 2.

2.2. Efficacy Data. Transition probabilities between model health states were derived from the survival and dialysis initiation endpoints from the INDEPENDENT-CKD trial which followed patients for a duration of 3 years or until death (Figures 2(a) and 2(b)) [16]. 


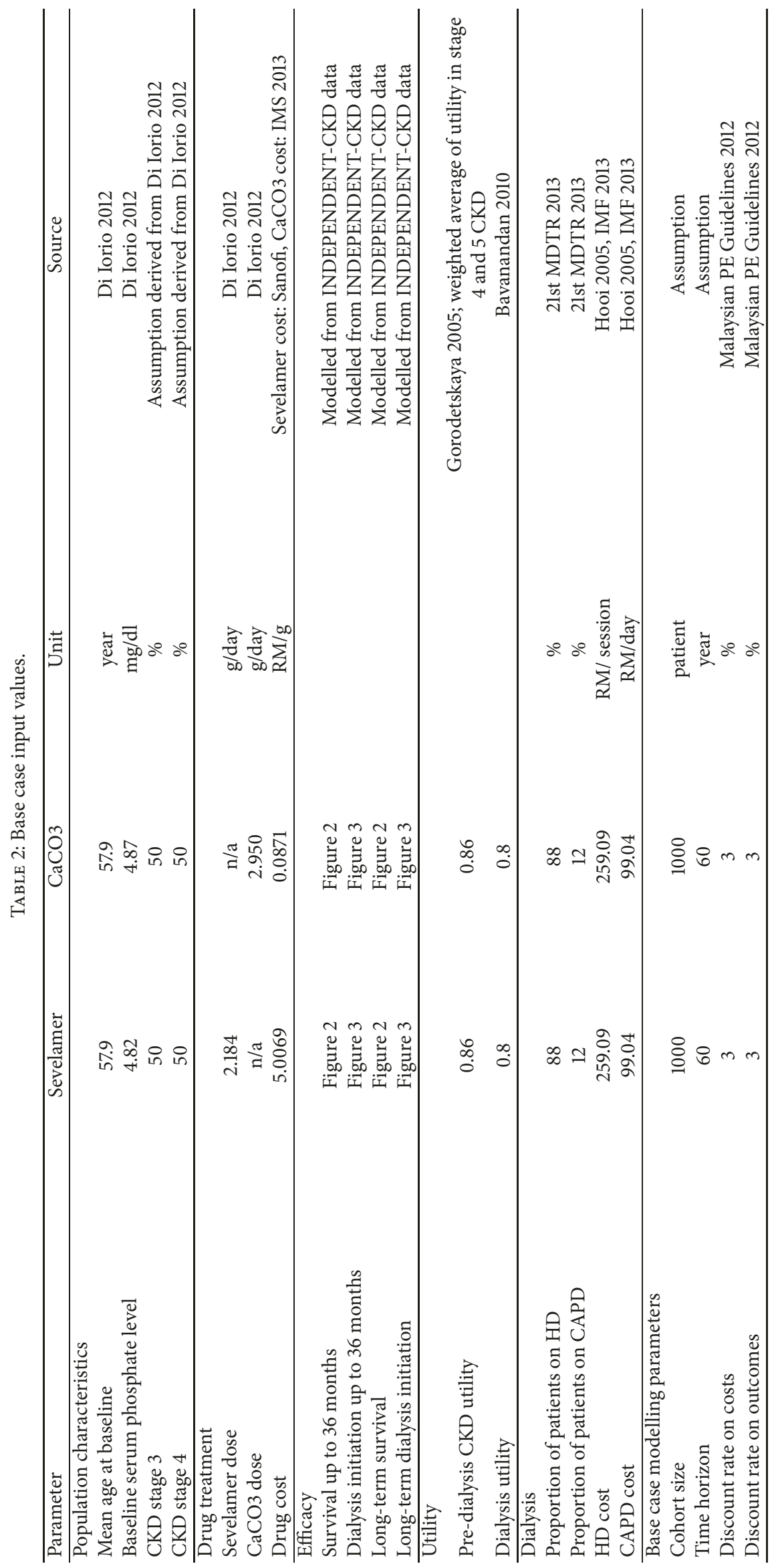




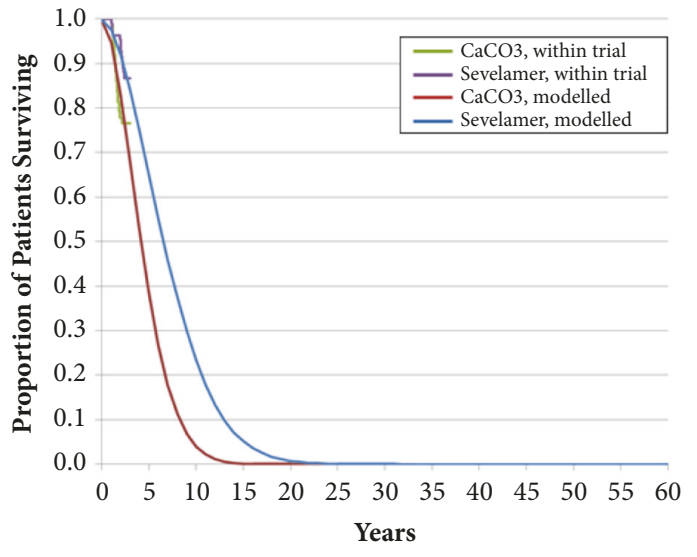

(a)

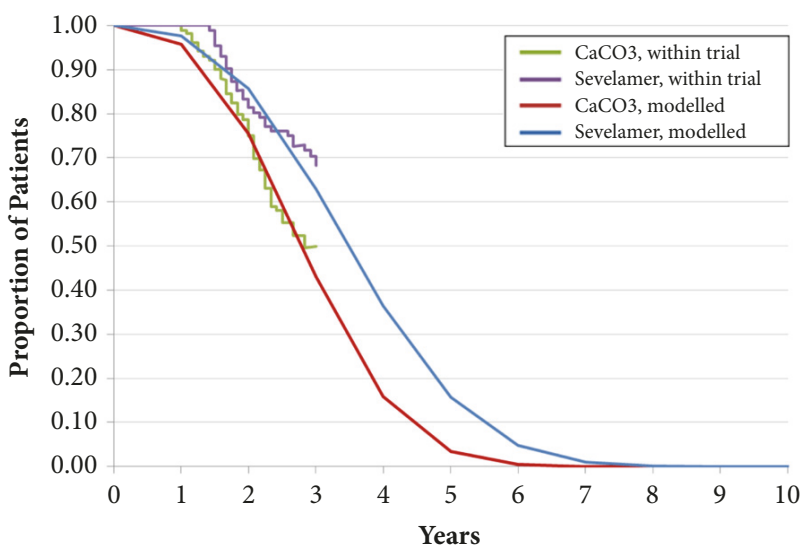

(b)

Figure 2: (a) Patient survival projected the from INDEPENDENT-CKD trial. Source: Di Iorio 2012, Cornerstone Research Group 2012. (b) Dialysis initiation projected from the INDEPENDENT-CKD trial. Source: Di Iorio 2012, Cornerstone Research Group 2012.

2.3. Health Utility Data. Health utility data inputs in the model required data for the dialysis and predialysis health states. For the quality of life (QOL) of dialysis, we used QOL data of Malaysian dialysis patients [23]. The utility score associated with a predialysis health state was based on a study by Gorodetskaya I et al. [24]. The base case utility input values are listed in Table 2.

2.4. Resource Use and Costs. The perspective of the analysis was that of the provider, i.e., Ministry of Health Malaysia $(\mathrm{MOH})$. Direct medical costs of drugs and dialysis incurred by the $\mathrm{MOH}$ were included in the analysis. The costs of other medical resources, i.e., hospitalization, concomitant drugs, treatment of adverse events (AE), and indirect costs (out-ofpocket expenses, productivity losses), were excluded from the analysis.

The medication costs of sevelamer and $\mathrm{CaCO} 3$ were calculated by multiplying the average daily drug dose reported in the INDEPENDENT-CKD trial with the latest available unit prices of sevelamer and generic $\mathrm{CaCO} 3$ in Malaysia. The cost per gram of sevelamer was derived from the indicative price offered by the drug manufacturer to the $\mathrm{MOH}$ whereas the cost of $\mathrm{CaCO} 3$ was obtained from the public sector cost of generic $\mathrm{CaCO} 3$ in the 3rd quarter of 2013 [25].

The cost of haemodialysis (HD) and continuous ambulatory peritoneal dialysis (CAPD) were obtained from a previous study of the $\mathrm{MOH}$ dialysis program conducted in 2001, adjusted to 2013 values by applying general inflation rates obtained from the International Monetary Fund [26, 27]. Dialysis costs were annualized assuming three HD sessions per week (156 HD sessions per year) and daily use of CAPD (365 CAPD days per year) according to the dialysis practice in Malaysia. The base case analysis inputs are listed in Table 2.

Currency conversions from RM to US\$ values presented in this study were calculated using the exchange rate on 31 December 2013 of $\$ 1$ to RM3.28 as the index year of study was 2013 [28].
2.5. Analysis. The model simulated the costs and outcomes over the lifetime of the entire cohort from initiation of CBB therapy to dialysis and/or death. Future costs and QALYs were discounted at 3\% per annum to the reference year as recommended by the Malaysian Pharmacoeconomics Guidelines [29]. Analysis was also performed using life years (LY) as health outcome.

We performed scenario sensitivity analysis by varying variables as recommended by guidelines and for key variables that may possibly change the conclusions that are drawn from the base case analysis. The variables that were analysed were discount rate, time horizon, drug dose, utility while on dialysis, and HD cost. As well, a probabilistic sensitivity analysis (PSA) was performed over 10,000 simulations to capture the uncertainty in several parameters in the model, namely, hazard ratios of survival and inception of dialysis, daily doses, and unit costs of sevelamer and $\mathrm{CaCO} 3$.

2.6. Assessment Criteria. According to the WHO guidelines, a treatment is cost-effective if the incremental costeffectiveness ratio (ICER) is below a maximum threshold of 3 times GDP per capita for a country when comparing one treatment against another [30]. The ICER, in this case, compares the cost and QALYs gained from treatment with sevelamer relative to $\mathrm{CaCO} 3[19,29,31,32]$. The ICER was calculated using the following formula:

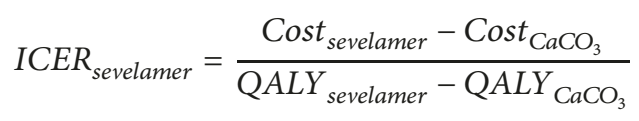

Based on the IMF's projected GDP per capita for Malaysia of RM33,132 in 2013, treatments with ICER below RM33,132 per QALY would be considered highly cost-effective, ICERs from RM33,132 to RM99,395 could be considered cost-effective while ICERs exceeding RM99,395 are not cost-effective [27, 30].

\section{Results}

3.1. Base Case Analysis. As shown in Table 3 the lifetime cost of treatment with sevelamer was higher at RM159,901 
TABLE 3: Base case results (3\% discount on costs and outcomes).

\begin{tabular}{lccccc}
\hline Treatment & $\begin{array}{c}\text { Lifetime cost per } \\
\text { patient (RM) }\end{array}$ & Effect (LY) & $\begin{array}{c}\text { ICER (Cost per LY } \\
\text { gained) }\end{array}$ & $\begin{array}{c}\text { Effect (QALY) } \\
\text { ICER (Cost per QALY } \\
\text { gained) }\end{array}$ & $\begin{array}{c}\text { (Rined } \\
\text { CaCO3 }\end{array}$ \\
Sevelamer & 159,139 & 4.25 & - & 3.54 & - \\
Incremental & 82,763 & 6.37 & - & 5.27 & - \\
\hline
\end{tabular}

TABLE 4: Scenario sensitivity analyses results.

\begin{tabular}{|c|c|c|c|c|}
\hline No. & Variable & Base case input value & Sensitivity input value & $\begin{array}{c}\text { ICER (Cost (RM) per } \\
\text { QALY gained) }\end{array}$ \\
\hline la & Undiscounted cost and outcomes & $3 \%$ & $0 \%$ & 48,033 \\
\hline $1 \mathrm{~b}$ & $5 \%$ discount rate & $3 \%$ & $5 \%$ & 47,419 \\
\hline $2 \mathrm{a}$ & 3-year time horizon & 60 years & 3 years & 25,438 \\
\hline $2 b$ & 10-year time horizon & 60 years & 10 years & 47,586 \\
\hline $3 \mathrm{a}$ & High sevelamer dose & $2.184 \mathrm{~g}$ & $4.8 \mathrm{~g}$ & 65,210 \\
\hline $3 b$ & High $\mathrm{CaCO} 3$ dose & $2.95 \mathrm{~g}$ & $7.5 \mathrm{~g}$ & 47,325 \\
\hline 4 & Low dialysis utility & 0.8 & 0.72 & 51,086 \\
\hline $5 a$ & Low HD cost & RM259.09 & RM197.48 & 40,627 \\
\hline $5 b$ & High HD cost & RM259.09 & RM350.00 & 58,084 \\
\hline 6 & Exclude dialysis cost & RM259.09 & 0 & 14,407 \\
\hline
\end{tabular}

$(\$ 48,750)$ compared to $\mathrm{RM77,139}(\$ 23,518)$ per patient on $\mathrm{CaCO} 3$. Yet, treatment with sevelamer gained more life years and QALY than CaCO3. In terms of life years, 6.37 years were gained on sevelamer compared to 4.25 years on $\mathrm{CaCO} 3$, whereas in terms of QALYs, sevelamer gained 5.27 versus 3.54 QALY gained using CaCO3. Hence, the ICER of treatment with sevelamer relative to $\mathrm{CaCO} 3$ was $\mathrm{RM} 39,050$ $(\$ 11,906)$ per LY gained and RM47,679 $(\$ 14,536)$ per QALY gained.

At RM47,679 per QALY gained, the ICER of treatment with sevelamer was between one to two times the estimated current Malaysian GDP per capita of RM33,132 and RM66,263, respectively. Based on WHO cost-effectiveness threshold, sevelamer would be considered cost-effective compared to $\mathrm{CaCO} 3$ for the treatment of hyperphosphatemia in CKD patients in Malaysia.

3.2. Scenario Sensitivity Analysis. In the scenario sensitivity analysis, the ICER of sevelamer compared to $\mathrm{CaCO} 3$ varied from RM14,407 to RM 65,210 per QALY gained, with the ICER being most sensitive to varying the cost of sevelamer (due to higher assumed daily dose of sevelamer), HD cost, and the time horizon. The results of scenario sensitivity analyses are shown in Table 4 .

Assuming a high sevelamer dose of $4.8 \mathrm{~g}$ per day would increase the ICER to RM65, 210 per QALY gained whereas assuming higher HD cost of RM350 per procedure would increase the ICER to RM 58,084 per QALY gained. On the other hand, excluding the cost of dialysis in the extended lifetime of the patient would reduce the ICER to RM 14,407. Overall, none of the one-way sensitivity scenarios exceeded the two times GDP per capita threshold of RM66,263 per QALY gained and sevelamer remained cost-effective. Reducing study duration to a 3-year time horizon lowered the ICER

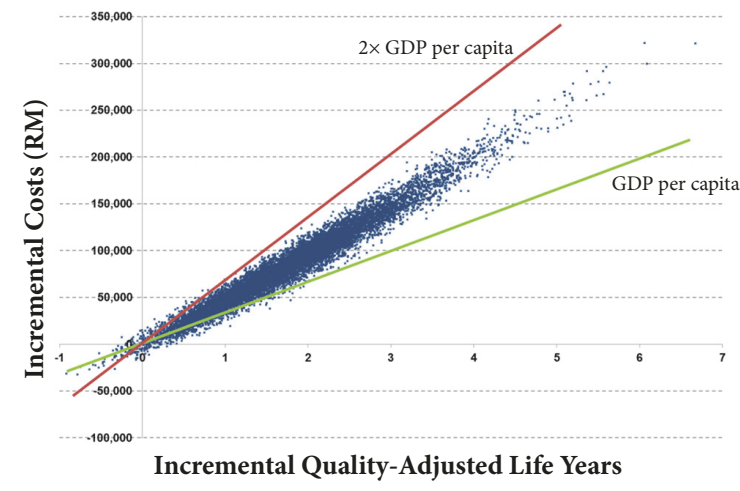

FIGURE 3: Scatter plot of ICER values generated from probabilistic sensitivity analysis.

and sevelamer becomes highly cost-effective at RM25,438 per QALY gained.

3.3. Probabilistic Sensitivity Analyses. Figure 3 illustrates the results of PSA in the form of an ICER scatterplot, which shows that, through 10,000 PSA simulations, the ICER values clustered in a narrow range around the base case ICER value of RM47,679 per QALY gained and between one and two times GDP per capita.

The cost-effectiveness acceptability curve shown in Figure 4 reaffirmed that in most simulations, the ICER would be between one and two times GDP per capita. The results of PSA showed that $98.9 \%$ of simulations generated ICERs below two times GDP per capita. Based on the PSA results, sevelamer would very likely be cost-effective for the treatment of hyperphosphatemia in CKD-ND patients in Malaysia as 


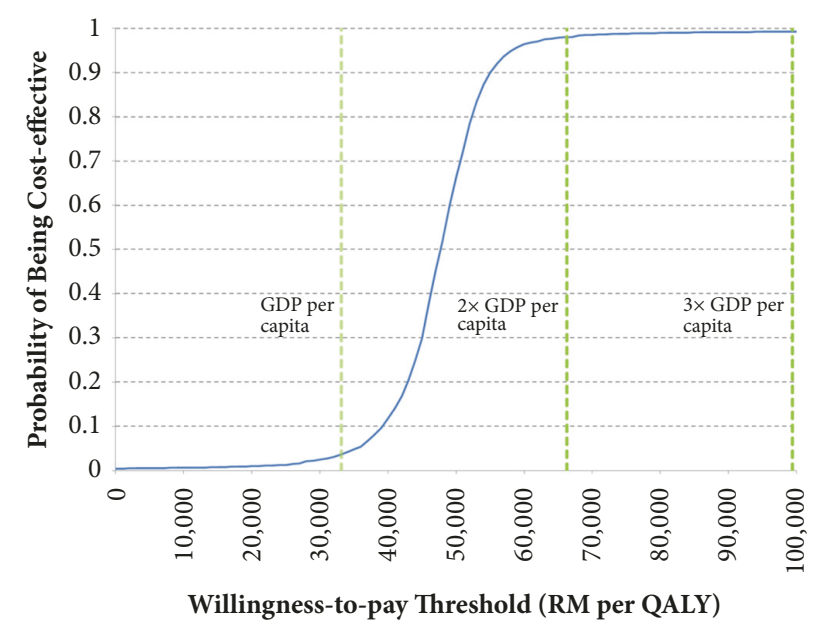

FIgURE 4: Cost-effectiveness acceptability curve.

it was unlikely that the ICER would exceed the WHO costeffectiveness threshold of three times GDP per capita.

\section{Discussion}

Our results are consistent with the earlier studies which demonstrated cost-effectiveness of sevelamer in UK and Italy $[17,18]$. The ICER is less than twice the GDP per capita for the country.

Our study has a number of strengths. To our knowledge, this study is the first attempt at an evaluation of the relative cost and benefits of sevelamer and $\mathrm{CaCO} 3$ in a predialysis population in a developing country setting. Secondly, we utilized Malaysian costs and utility data as inputs where possible. Thirdly, sensitivity analyses were conducted with both scenario and probabilistic sensitivity analyses. The PSA predicted that just $0.4 \%$ of simulations would exceed the WHO cost-effectiveness threshold, reinforcing the robustness of the base case findings that sevelamer is cost-effective.

We also note several limitations to the study. Firstly, the analysis was performed using a global cost-effectiveness decision model which comprised only three health states and was based on data from the INDEPENDENT-CKD trial [16]. There was also limited flexibility for customization and longterm efficacy which was based on 36-month follow-up data extrapolated to lifetime by regression modelling. However, as an independent study with a randomized, controlled trial design, it provided a possibly less biased comparative efficacy data from a matched population and remains the best available data in the absence of other head-to-head comparisons of sevelamer and $\mathrm{CaCO} 3$ in the local setting.

This study was technically designed to assess costeffectiveness against the WHO threshold, but it may also be useful to compare our result against previous funding decisions by the Malaysian $\mathrm{MOH}$. Of relevance is a comparison to haemodialysis treatment provided by $\mathrm{MOH}$ which has been estimated to cost RM33,642 per annum in 2001 in a previous study [26]. Factoring for inflation and exchange rate changes since 2001, we estimated the current cost of haemodialysis to be RM44,138 per patient per year [26, 28], which is slightly higher than the ICER of sevelamer in the present study at RM39,050 per LY. This means that increasing expenditure on a medication like sevelamer could prevent patients from going on to dialysis, which is a favourable clinical outcome as well as save costs through over the lifetime of patients. Beyond being a good clinical outcome, delay of dialysis treatment would also allow patients with early renal insufficiency to lead better quality and healthier lives which can be defined as priceless to an individual patient. From a societal point of view, renal patients who are not on dialysis have greater potential to contribute fully to the society through their familial, social, and economic contributions.

\section{Conclusions}

In conclusion, our analysis indicates that sevelamer can be a cost-effective treatment for hyperphosphatemia in Malaysian CKD-ND patients compared to $\mathrm{CaCO} 3$ at an ICER of RM47,679 per QALY gained. Results of sensitivity analyses did not substantially differ from the base case results and indicated that the base case results were robust and did not exceed the CE threshold of three times GDP per capita per QALY gained. Further studies incorporating long-term efficacy data, local utilities, cardiovascular disease effects, and adverse events may improve the precision of the results, but we anticipate it would be unlikely to change the overall conclusion. Our results for Malaysia suggest that sevelamer may be cost-effective in middle-income countries. This study finds that sevelamer is potentially cost-effective compared to $\mathrm{CaCO} 3$, for the treatment of hyperphosphatemia in predialysis CKD III-V. We propose that sevelamer should be an option in the treatment of Malaysian predialysis patients with hyperphosphatemia, particularly those with high calcium load. However, definitive conclusions about the cost-effectiveness of sevelamer should be confirmed through individual country-level studies incorporating local data.

\section{Data Availability}

The data supporting the findings of this study are stated clearly and available in the manuscript.

\section{Ethical Approval}

This study was registered and approved by the Ethics Committee of the National Medical Research Register.

\section{Disclosure}

However, apart from providing the indicative price of sevelamer in Malaysia, the sponsor did not have any influence over the data collection, analysis, interpretation of data, and manuscript writing.

\section{Conflicts of Interest}

The authors declare that there are no conflicts of interest regarding the publication of this article. 


\section{Acknowledgments}

This study received Grant NMRR-11-24-8143 from National Institute of Health Malaysia.

\section{References}

[1] L. S. Hooi, L. M. Ong, G. Ahmad et al., "A population-based study measuring the prevalence of chronic kidney disease among adults in West Malaysia," Kidney International, vol. 84, no. 5, pp. 1034-1040, 2013.

[2] A. Ramachandran, C. Snehalatha, A. S. Shetty, and A. Nanditha, "Trends in prevalence of diabetes in Asian countries," World Journal of Diabetes, vol. 3, no. 6, pp. 110-117, 2012.

[3] C. L. Tam, G. Bonn, S. H. Yeoh, and C. P. Wong, "Investigating diet and physical activity in Malaysia: education and family history of diabetes relate to lower levels of physical activity," Frontiers in Psychology, vol. 5, 2014.

[4] Q. Zhang and D. Rothenbacher, "Prevalence of chronic kidney disease in population-based studies: systematic review," $B M C$ Public Health, vol. 8, article 117, 2008.

[5] V. Jha, G. Garcia-Garcia, K. Iseki et al., "Chronic kidney disease: global dimension and perspectives," The Lancet, vol. 382, no. 9888, pp. 260-272, 2013.

[6] T.-O. Lim, A. Goh, Y.-N. Lim, Z. M. M. Zaher, and A. B. Suleiman, "How public and private reforms dramatically improved access to dialysis therapy in Malaysia," Health Affairs, vol. 29 , no. 12 , pp. 2214-2222, 2010.

[7] B. L. Goh and H. S. Wong, "24th Report of the Malaysian Dialysis and Transplant Registry," 2016.

[8] C.-Y. Hsu and G. M. Chertow, "Elevations of serum phosphorus and potassium in mild to moderate chronic renal insufficiency," Nephrology Dialysis Transplantation, vol. 17, no. 8, pp. 1419$1425,2002$.

[9] M. Kanbay, D. Goldsmith, A. Akcay, and A. Covic, "Phosphate - The silent stealthy cardiorenal culprit in all stages of chronic kidney disease: A systematic review," Blood Purification, vol. 27, no. 2, pp. 220-230, 2009.

[10] A. Bellasi, M. Mandreoli, L. Baldrati et al., "Chronic kidney disease progression and outcome according to serum phosphorus in mild-to-moderate kidney dysfunction," Clinical Journal of the American Society of Nephrology, vol. 6, no. 4, pp. 883-891, 2011.

[11] KDIGO, "KDIGO clinical practice guideline for the diagnosis, evaluation, prevention, and treatment of Chronic Kidney Disease-Mineral and Bone Disorder (CKD-MBD)," Kidney International Supplements, vol. S1, no. 130, 2009.

[12] G. M. Chertow, S. K. Burke, and P. Raggi, "Sevelamer attenuates the progression of coronary and aortic calcification in hemodialysis patients," Kidney International, vol. 62, no. 1, pp. 245-252, 2002.

[13] S. K. Burke, M. A. Dillon, D. E. Hemken, M. S. Rezabek, and J. M. Balwit, "Meta-analysis of the effect of sevelamer on phosphorus, calcium, PTH, and serum lipids in dialysis patients," Advances in Chronic Kidney Disease, vol. 10, no. 2, pp. 133-145, 2003.

[14] D. Russo, I. Miranda, C. Ruocco et al., "The progression of coronary artery calcification in predialysis patients on calcium carbonate or sevelamer," Kidney International, vol. 72, no. 10, pp. 1255-1261, 2007.

[15] M. J. Bolland, A. Grey, A. Avenell, G. D. Gamble, and I. R. Reid, "Calcium supplements with or without vitamin D and risk of cardiovascular events: reanalysis of the Women's Health Initiative limited access dataset and meta-analysis," British Medical Journal, vol. 342, no. 7804, Article ID d2040, 2011.

[16] B. Di Iorio, A. Bellasi, and D. Russo, "Mortality in kidney disease patients treated with phosphate binders: A randomized study," Clinical Journal of the American Society of Nephrology, vol. 7, no. 3, pp. 487-493, 2012.

[17] M. Thompson, S. Bartko-Winters, L. Bernard, A. Fenton, C. Hutchison, and B. Di Iorio, "Economic evaluation of sevelamer for the treatment of hyperphosphatemia in chronic kidney disease patients not on dialysis in the United Kingdom," Journal of Medical Economics, vol. 16, no. 6, pp. 744-755, 2013.

[18] M. Ruggeri, F. Cipriani, A. Bellasi, D. Russo, and B. D. Iorio, "Sevelamer is cost-saving vs. calcium carbonate in nondialysis-dependent CKD patients in Italy: A patient-level costeffectiveness analysis of the INDEPENDENT study," Blood Purification, vol. 37, no. 4, pp. 316-324, 2014.

[19] M. F. Drummond, G. L. Stoddart, and G. W. Torrance, Methods for the economic evaluation of health care programmes, Oxford University Press, Oxford, UK, 1997.

[20] A. Gray, Applied Methods of Cost-Effectiveness Analysis in Health Care, Oxford University Press, Oxford, UK, 2011.

[21] M. J. Taylor, H. A. Elgazzar, S. Chaplin, D. Goldsmith, and D. A. Molony, "An economic evaluation of sevelamer in patients new to dialysis," Current Medical Research and Opinion, vol. 24, no. 2, pp. 601-608, 2008.

[22] J. H. S. You, W.-K. Ming, W.-A. Lin, and Y.-H. Tarn, "Early supplemented low-protein diet restriction for chronic kidney disease patients in Taiwan - A cost-effectiveness analysis," Clinical Nephrology, vol. 84, no. 4, pp. 189-197, 2015.

[23] S. Bavanandan, S. Azmi, and A. Goh, "Quality of life of Malaysian dialysis patients on transplant waiting list," in Proceedings of the ISPOR 4th Asia-Pacific Conference, Phuket, Thailand, 2010.

[24] I. Gorodetskaya, S. Zenios, C. E. McCulloch et al., "Healthrelated quality of life and estimates of utility in chronic kidney disease," Kidney International, vol. 68, no. 6, pp. 2801-2808, 2005.

[25] IMS Health, Malaysian Pharmaceutical Audit, Sanofi Aventis, 2013.

[26] L. S. Hooi, T. O. Lim, A. Goh et al., "Economic evaluation of centre haemodialysis and continuous ambulatory peritoneal dialysis in Ministry of Health hospitals, Malaysia," Nephrology, vol. 10, no. 1, pp. 25-32, 2005.

[27] Fund IM, World Economic Outlook Database, 2013, https://www .imf.org/external/pubs/ft/weo/2013/02/weodata/index.aspx.

[28] “Bank Negara Malaysia, Exchange Rates," http://www.bnm.gov .my/index.phpAvailable at ?ch=statisticpg=stats_exchangerates.

[29] Pharmaceutical Services Division (Ministry of Health Malaysia), "Pharmacoeconomic Guideline for Malaysia," 2012.

[30] Pharmacoeconomic Guideline for Malaysia, "Cost effectiveness threshold," http://www.who.int/choice/costs/CER_thresholds/ en/.

[31] D. Caldwell, "Decision Modelling for Health Economic Evaluation. A Briggs, M Sculpher, K Claxton," International Journal of Epidemiology, vol. 36, no. 2, pp. 476-477, 2007.

[32] J. J. Caro, A. H. Briggs, U. Siebert, and K. M. Kuntz, "Modeling good research practices - Overview: A report of the ISPORSMDM modeling good research practices task force-1," Value in Health, vol. 15, no. 6, pp. 796-803, 2012. 


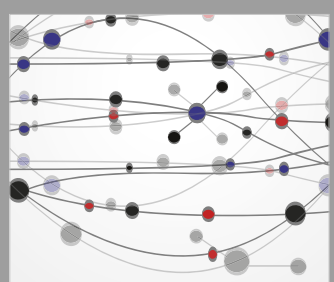

The Scientific World Journal
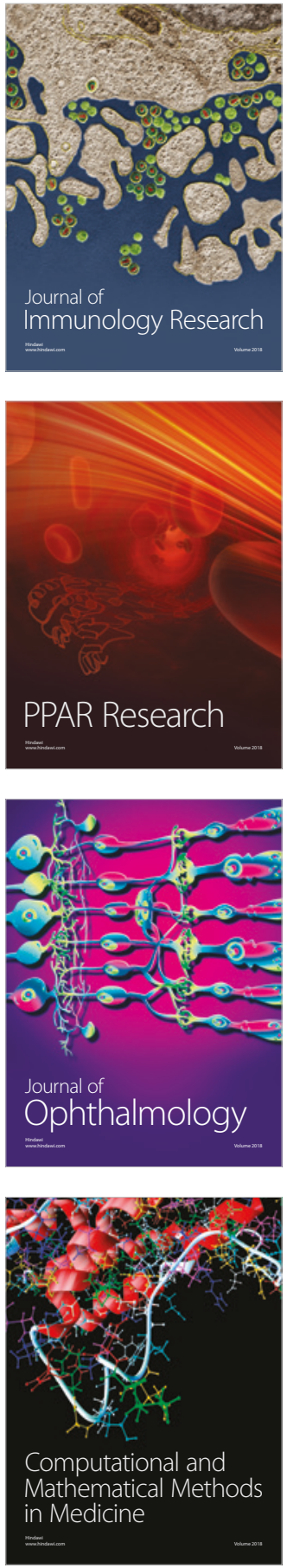

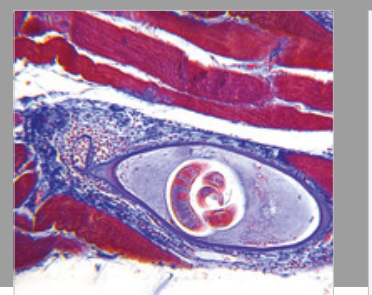

Gastroenterology Research and Practice

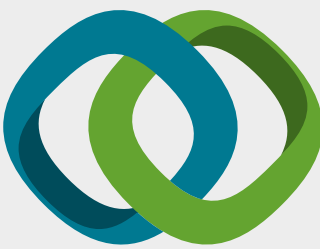

\section{Hindawi}

Submit your manuscripts at

www.hindawi.com
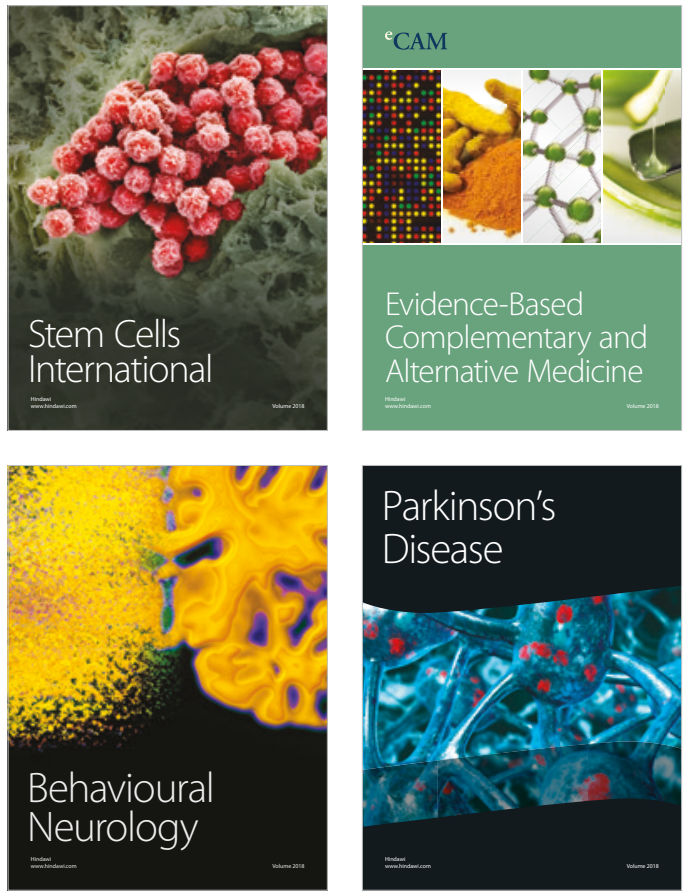

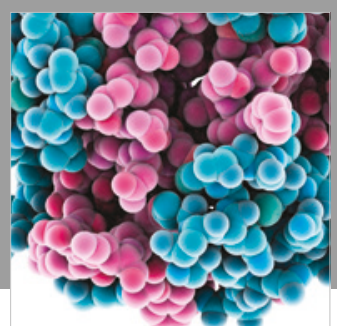

ournal of

Diabetes Research

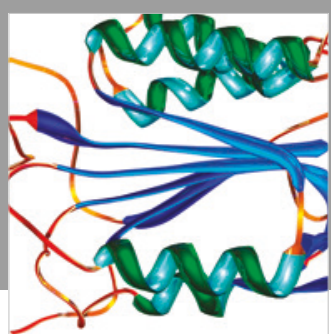

Disease Markers
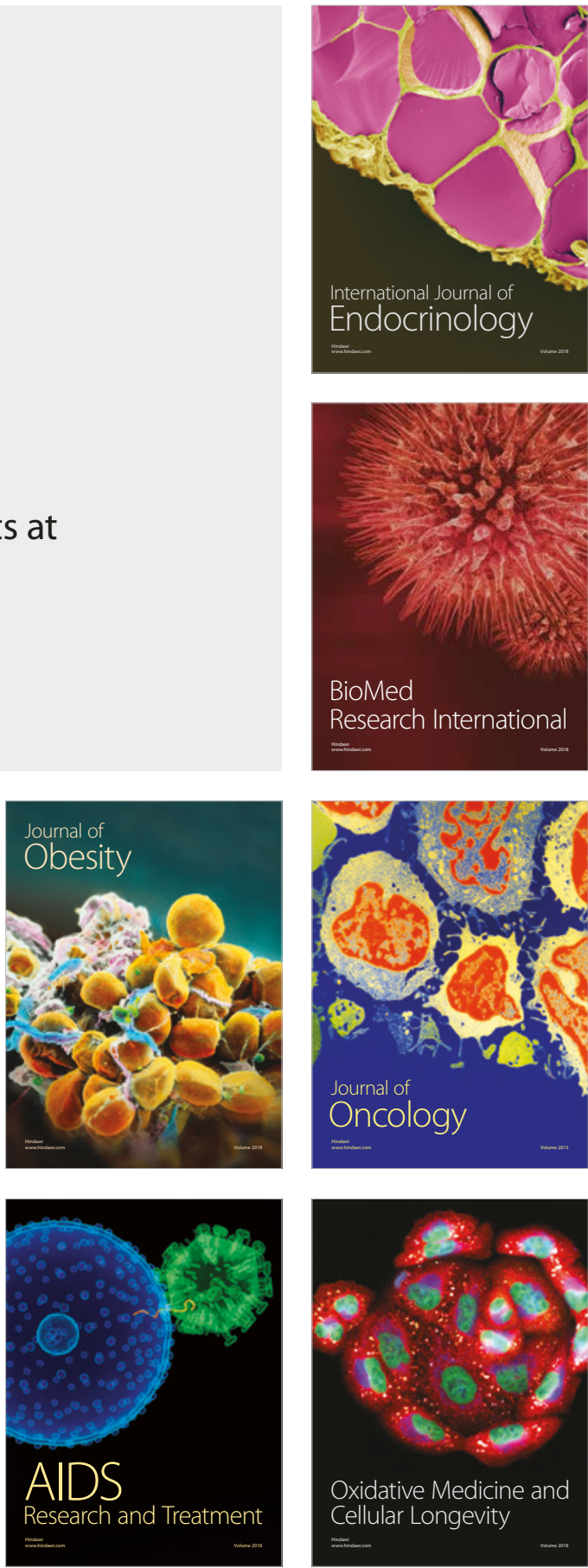